\title{
Abrupt Intralesional Color Change on Dermoscopy as a New Indicator of Early Superficial Spreading Melanoma in a Japanese Woman
}

\author{
Anna Sadayasu $^{\mathrm{a}}$ Masaru Tanaka $^{\mathrm{a}}$ Yoshifumi Maumi $^{\mathrm{a}}$ \\ Eriko Ikeda $^{a} \quad$ Mizuki Sawada $^{a}$ Sumiko Ishizaki ${ }^{a}$ Yoshiyuki Murakami ${ }^{c}$ \\ Mariko Fujibayashi ${ }^{b}$ \\ Departments of ${ }^{a}$ Dermatology and ${ }^{b}$ Pathology, Tokyo Women's Medical University \\ Medical Center East, and 'Mildix Skin Clinic, Tokyo, Japan
}

\section{Key Words}

Superficial spreading melanoma $\cdot$ Dermoscopy $\cdot$ Structureless area

\begin{abstract}
Diagnosis of superficial spreading melanoma in the early stage is often difficult, even with dermoscopy. We report the case of a 37-year-old Japanese woman with superficial spreading melanoma in her left buttock. The lesion developed 20 years before becoming visible and gradually enlarged over the past few years without any symptoms. Physical examination showed a well-demarcated dark-brown macule $10 \mathrm{~mm}$ in diameter. Dermoscopy demonstrated a central dark area with a blue-grey structureless area, a milky-red area with irregular blue-grey dots or globules suggestive of regression structures, and multifocal black pigmentation with whitish scaly areas. An abrupt intralesional change in color from a central dark area to a peripheral light-brown area was also seen. The peripheral area showed an atypical pigment network with an obscure mesh and holes. Histopathologic examination of the lesion showed acanthosis with melanocytic proliferation and nuclear atypia, a band-like lymphocytic infiltrate, melanophages and a few nests of melanocytes just beneath the epidermis. The epidermal melanocytes were positive for S-100, Melan-A and HMB-45, but the dermal nests of melanocytes were negative for HMB-45 and positive for S-100 and Melan-A. A diagnosis of superficial spreading melanoma with a tumor thickness of $0.4 \mathrm{~mm}$ (pT1aNOM0, stage 1A) was established based on the clinical, dermoscopic and histopathologic findings. This case
\end{abstract}

KARGER 125:s $\quad \begin{aligned} & \text { Anna Sadayasu, MD } \\ & \text { Department of Dermatology } \\ & \text { Tokyo Women's Medical University Medical Center East } \\ & \text { 2-1-10 Nishi-Ogu, Arakawa-ku, Tokyo 116-8567 (Japan) } \\ & \text { E-Mail u.anna.1224@gmail.com }\end{aligned}$


Sadayasu et al.: Abrupt Intralesional Color Change on Dermoscopy as a New Indicator of Early Superficial Spreading Melanoma in a Japanese Woman

suggests that dermoscopy is useful in the diagnosis of this condition. An abrupt intralesional change of color might be a new indicator of early superficial spreading melanoma.

(c) 2015 S. Karger AG, Basel

\section{Introduction}

Superficial spreading melanoma simulates various skin lesions such as seborrheic keratosis [1], pigmented basal cell carcinoma [2] and pigmented Bowen's disease [3-5] in addition to melanocytic nevus [6]. We report a case of superficial spreading melanoma in a Japanese woman that showed a potential relationship between dermoscopic and histopathologic findings.

\section{Case Presentation}

A 37-year-old Japanese woman presented to our clinic with a dark-brown skin lesion on the left buttock. Her family history and medical history were negative for melanoma. The skin lesion had first been noticed 20 years previously and had gradually enlarged over the past few years. Physical examination showed a well-demarcated, dark-brown macule on the left buttock measuring $10 \mathrm{~mm}$ in diameter (fig. 1). Dermoscopy demonstrated a central dark area with a blue-grey structureless area, a milky-red area with irregular blue-grey dots or globules suggestive of regression structures, and multifocal black pigmentation with whitish scales. The region also showed an abrupt intralesional change in color from a central dark area to a peripheral light-brown area. The peripheral area showed an atypical pigment network with an obscure mesh and holes (fig. 2).

Histopathologic examination of the lesion showed mild acanthosis with melanocytic proliferation and nuclear atypia, a band-like lymphocytic infiltrate, melanophages and a few nests of melanocytes just beneath the epidermis (fig. 3a). There were atypical melanocytes with large nuclei mainly at the basal layer, but some of the cells were in the upper layer of the epidermis in the center of the lesion (fig. 3b). There were also some nests of melanocytes with large nuclei in the subpapillary dermis (fig. 3c). Furthermore, there was proliferation of individual atypical melanocytes and an uneven deposition of melanin in the basal layer as well as the suprabasal layer at the periphery (fig. 3d). Atypical melanocytes in the epidermis were positive for Melan-A, HMB-45 and S-100. Dermal nests of atypical melanocytes were only positive for Melan-A and S-100, but negative for HMB-45 (fig. 4). No antecedent nevus cell was seen in the entire specimen. Based on the clinical, dermoscopic and histopathologic findings, a diagnosis of superficial spreading melanoma with a tumor thickness of $0.4 \mathrm{~mm}$ (pT1aN0M0, stage 1A) was established.

\section{Discussion}

The diagnosis of superficial spreading melanoma is difficult in the early stage, even with dermoscopy. When the size of the lesion is small, the irregular proliferation of melanocytes and uneven distribution of melanin granules are not prominent. One of the methods used for the early diagnosis of melanoma is digital dermoscopy [7, 8], especially through the dermoscopic visualization of the 'ugly duckling' sign $[9,10]$. However, melanocytic nevi can also become enlarged during the early stages; therefore, size alone cannot distinguish melanoma 
Sadayasu et al.: Abrupt Intralesional Color Change on Dermoscopy as a New Indicator of Early Superficial Spreading Melanoma in a Japanese Woman

from melanocytic nevi. Therefore, identifying an indicator for melanoma at an early stage is important.

Indicators such as an atypical pigment network, irregular dots or globules, and irregular streaks have already been identified, but inability to visualize these structures makes diagnosis difficult. Although the present case to some extent showed an atypical pigment network, through a thick mesh structure and small holes, in which the width of the mesh was thicker than the diameter of the holes, the color of the pigment network was entirely brownish with a slight shade disparity. The present case also showed an abrupt intralesional change in color from a blue-grey structureless area in the center to a peripheral light-brown pigment network, which we strongly suspected to be indicative of melanoma.

The use of dermoscopy for this case appeared to be extremely useful, especially for the diagnosis of the lesion that developed in the early stage of melanoma. Furthermore, an abrupt intralesional change in color might be a new indicator of early melanoma.

\section{Statement of Ethics}

The authors state that the patient gave her informed consent. The research complies with all ethical guidelines for human studies.

\section{Disclosure Statement}

The authors declare no conflict of interest. There was no funding.

\section{References}

1 Izikson L, Sober AJ, Mihm MC Jr, Zembowicz A: Prevalence of melanoma clinically resembling seborrheic keratosis: analysis of 9,204 cases. Arch Dermatol 2002;138:1562-1566.

2 White EA, Rabinovitz HS, Greene RS, Oliviero M, Kopf A: Pigmented basal cell carcinoma simulating melanoma in a burn scar. Cutis 2003;71:404-406.

3 Stante M, de Giorgi V, Massi D, Chiarugi A, Carli P: Pigmented Bowen's disease mimicking cutaneous melanoma: clinical and dermoscopic aspects. Dermatol Surg 2004;30:541-544.

4 Inoue T, Kobayashi K, Sawada M, Ishizaki S, Ito H, Fujibayashi M, Tanaka M: Dermoscopic features of pigmented Bowen's disease in a Japanese female mimicking malignant melanoma. Dermatol Res Pract 2010 DOI: $10.1155 / 2010 / 543091$.

5 Hayashi Y, Tanaka M, Suzaki R, Mori N, Konohana I: Dermoscopy of pigmented Bowen's disease mimicking early superficial spreading melanoma. Case Rep Dermatol 2009;1:11-15.

6 Makino E, Uchida T, Matsushita Y, Inaoki M, Fujimoto W: Melanocytic nevi clinically simulating melanoma. J Dermatol 2007;34:52-55.

7 Kittler H, Binder M: Follow-up of melanocytic skin lesions with digital dermoscopy: risks and benefits. Arch Dermatol 2002;138:1379.

8 Kittler H, Binder M: Risks and benefits of sequential imaging of melanocytic skin lesions in patients with multiple atypical nevi. Arch Dermatol 2001;137:1590-1595.

9 Grob JJ, Bonerandi JJ: The 'ugly duckling' sign: identification of the common characteristics of nevi in an individual as a basis for melanoma screening. Arch Dermatol 1998;134:103-104.

10 Inskip M, Magee J, Weedon D, Rosendahl C: When algorithms falter: a case report of a very small melanoma excised due to the dermatoscopic 'ugly duckling' sign. Dermatol Pract Concept 2013;3:59-62.

This case was presented at the 29th Annual Meeting of the Japanese Skin Cancer Society. 


\section{Case Reports in Dermatology}

Sadayasu et al.: Abrupt Intralesional Color Change on Dermoscopy as a New Indicato of Early Superficial Spreading Melanoma in a Japanese Woman

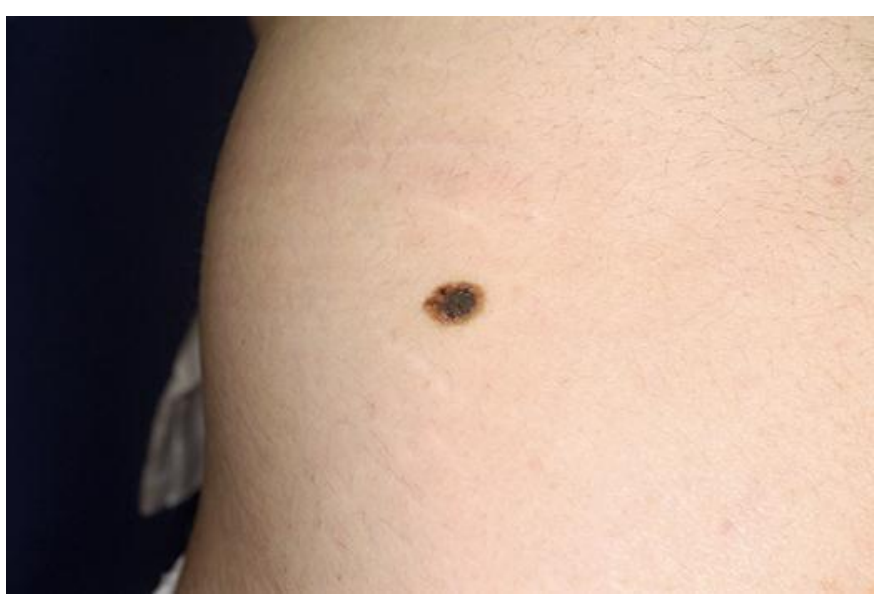

Fig. 1. Clinical photographs. Physical examination showed a well-demarcated, dark-brown macule $10 \mathrm{~mm}$ in diameter on the left buttock.

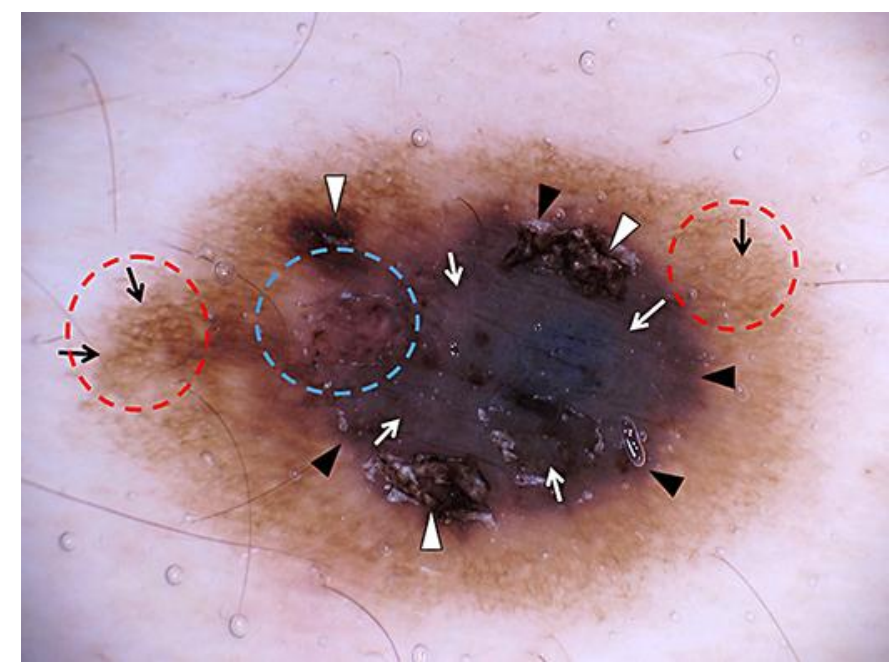

Fig. 2. Dermoscopic findings. Dermoscopy demonstrated a central dark area with a blue-grey structureless area (white arrows) and a milky-red area with irregular blue-grey dots or globules, which was suggestive of regression structures (blue circle). Multifocal black pigmentation with whitish scaly areas (white arrowheads) was also seen. The region also showed an abrupt intralesional change in color from a central dark area to a peripheral light-brown area (black arrowheads). The peripheral area showed an atypical thick pigment network (black arrows) with an obscure mesh and holes (red circles). 
Sadayasu et al.: Abrupt Intralesional Color Change on Dermoscopy as a New Indicator of Early Superficial Spreading Melanoma in a Japanese Woman
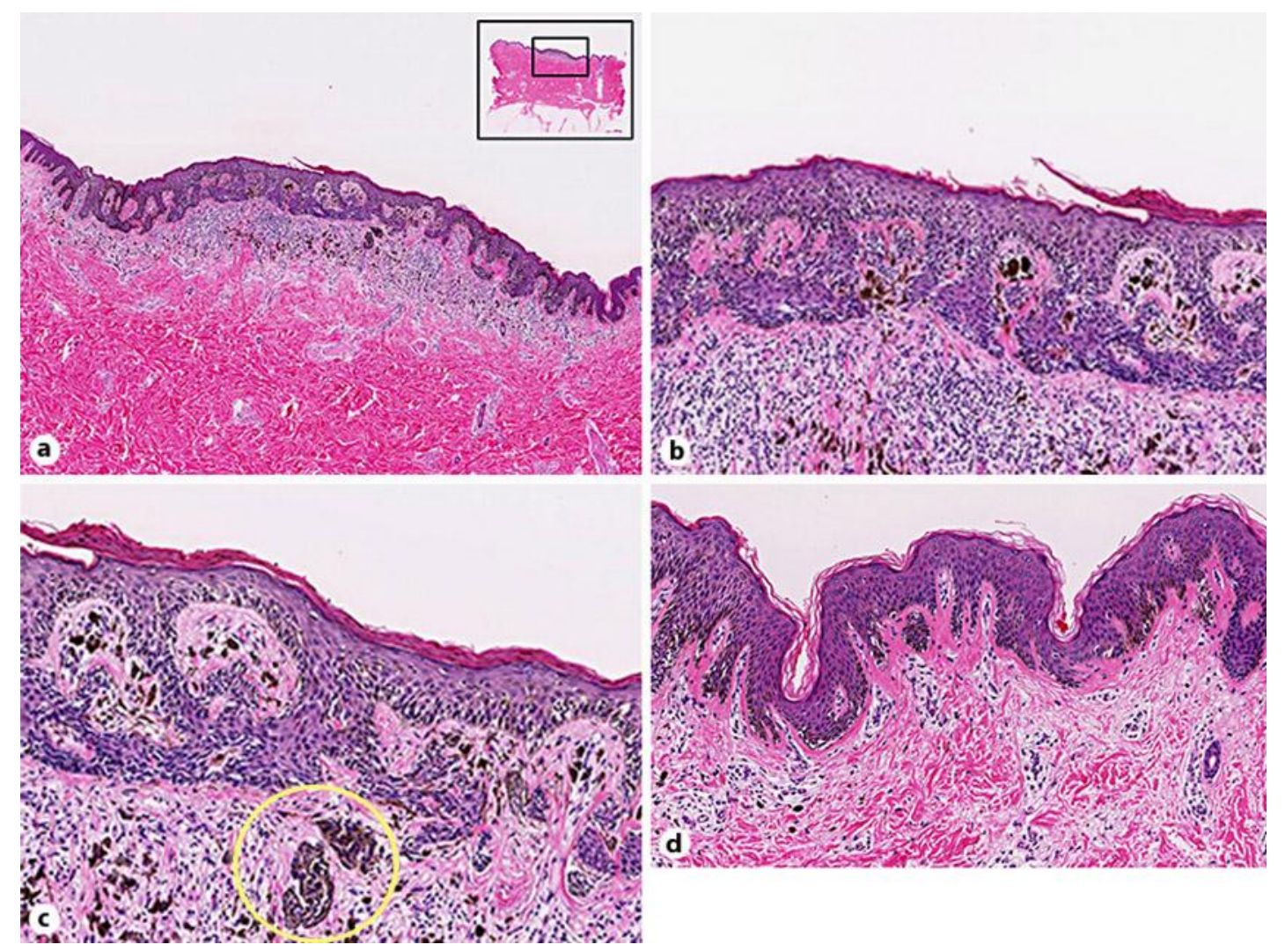

Fig. 3. Histopathologic findings. a Mild acanthosis and an irregular distribution of melanin deposition in the epidermis and the dermis were seen in the low-power view. Epidermal melanin deposition was more prominent at the periphery and less prominent in the center. Furthermore, a band-like cellular infiltrate intermingled with melanophages was seen in the subpapillary dermis. b Atypical melanocytes with large nuclei were mainly seen at the basal layer, but some of the cells in the upper layer of the epidermis lay in the center of the lesion. c Some nests of melanocytes with large nuclei (yellow circle) were seen in the subpapillary dermis. $\mathbf{d}$ Proliferation of individual atypical melanocytes and an uneven deposition of melanin were seen in the basal layer as well as in the suprabasal layer at the periphery. 


\section{Case Reports in Dermatology}

\begin{tabular}{l|l}
\hline Case Rep Dermatol 2015;7:123-128 \\
\hline DOI: 10.1159/000432391 & $\begin{array}{l}\text { C 2015 S. Karger AG, Basel } \\
\text { www.karger.com/cde }\end{array}$ \\
\hline
\end{tabular}

Sadayasu et al.: Abrupt Intralesional Color Change on Dermoscopy as a New Indicato of Early Superficial Spreading Melanoma in a Japanese Woman
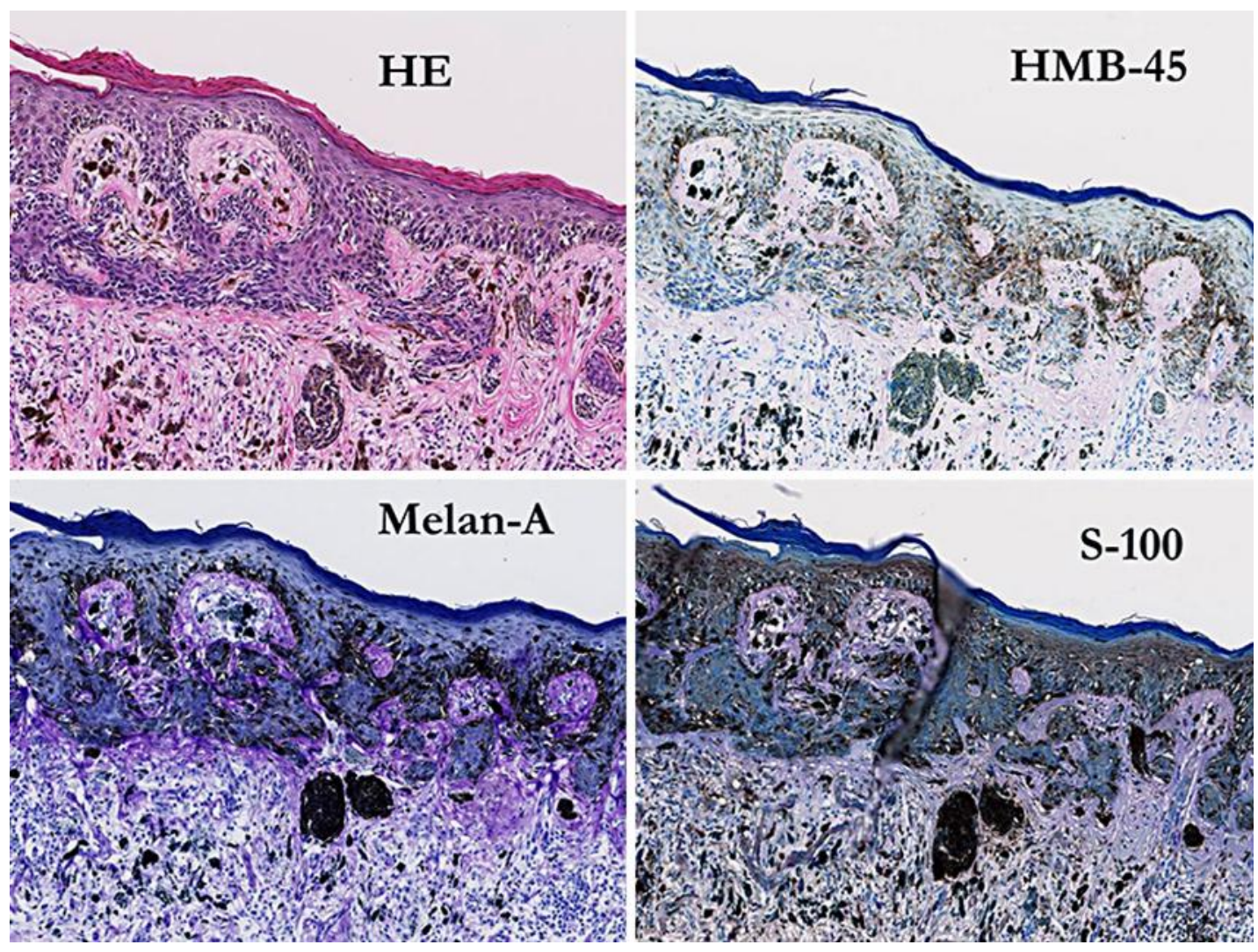

Fig. 4. Immunohistochemical findings. Atypical melanocytes in the epidermis were positive for Melan- $A$ HMB-45 and S-100. Dermal nests of atypical melanocytes were only positive for Melan-A and S-100, but negative for HMB-45. The tumor was $0.4 \mathrm{~mm}$ thick at the region of invasion. 